\title{
Néologismes et calques dans le kabyle moderne : Cas du code de la route et des journaux d'information
}

\begin{abstract}
Résumé
Notre contribution s'articule autour d'une analyse lexico-sémantique et syntaxique de la terminologie du code de la route d'Ali Khalfa (2005) et des journaux d'information en berbère (kabyle) en mettant en exergue les procédés utilisés lors de la création des néologismes et les caractéristiques syntaxiques de la langue utilisée. Pour ce faire, nous avons comparé la langue utilisée dans ces publications et celle de l'usage pour voir le degré d'attestation des règles en question dans le kabyle de l'usage moderne. Notre hypothèse consiste à dire que les textes de l'usage moderne du kabyle sont caractérisés par des structures calquées sur la langue française, et sur le plan de la création néologique et sur le niveau syntaxique. En effet, certaines créations néologiques par calque génèrent de nouvelles structures non attestées et incompréhensibles dans l'usage quotidien.
\end{abstract}

Mots-clés : lexique, syntaxe, néologie, calque, terminologie, usage.

\section{Summary}

Our contribution articulates around a lexico-semantic and syntactic analysis of the terminology of the traffic rules by Ali Khalfa ( 2005 ) and Berber (kabylian) newspapers, highlighting the processes used in the creation of neologisms and the syntactic characteristics of the language used. Within this intention, we compared the language used in these publications and that really in use in 
everyday life in order to check the degree of attestation of the rules in question. Our hypothesis consists in saying that the texts of the modern use are characterized by structures modeled on the French language both at the neological creation level as well as on that of syntactic one. Indeed, certain neological creations by layer generate new unattested and incomprehensible structures in the daily use.

Keywords: lexicon, syntax, neology, layer, terminology, use.

\section{Introduction}

À côté du français et de l'arabe, réservés aux domaines de l'enseignement, de l'administration et de la communication, le berbère (kabyle) - réservé depuis des millénaires aux domaines d'usages oraux, restreints à la vie quotidienne, longtemps mis à l'écart, souffre-douleur de tant de berbérophones qui peinent à le maintenir en survie - a bénéficié récemment d'une avancée remarquable en matière de statut institutionnel et constitutionnel ${ }^{1}$ et s'étend à de nouveaux domaines d'usage (enseignement, communication, etc.).

Par ailleurs, l'introduction du berbère dans les domaines officiels s'est faite sous la pression sociale. En effet, deux départements de langue et culture amazighes ont été créés par les luttes de ses locuteurs, l'un à l'Université de Tizi-Ouzou (1990), l'autre à l'Université de Bejaia (1991). Durant l'année scolaire 1994-1995, le boycott scolaire a permis l'introduction du berbère dans le système éducatif et la création du Haut-Commissariat à l'A mazighité (HCA), chargé de la réhabilitation de la langue et de la culture amazighes. En 1996, avec le projet de la révision de la constitution, le berbère est intégré, dans son préambule dans les constantes nationales à côté de l'islamité et de l'arabité. En 2001, avec les événements tragiques de la Kabylie, la langue berbère est reconnue en tant que deuxième langue nationale à côté de l'arabe, et ce lors de l'amendement de la constitution du 8 avril 2002. Dans la même conjoncture, en décembre 2003, la langue berbère a bénéficié d'un centre national pédagogique et linguistique pour l'enseignement du berbère, placé sous la tutelle du Ministère de l'Éducation Nationale. Dans le même processus, l'Université a bénéficié de l'ouverture de deux nouveaux

1 Consacré comme langue nationale dans la constitution algérienne en 2002 et langue nationale officielle en 2016. 
départements, l'un à l'Université de Bouira (2008), l'autre à l'Université de Batna (2012). Avec la révision de la constitution en 2016, le berbère a atteint le statut de langue nationale et officielle à côté de l'arabe. Durant la rentrée universitaire 2016-2017, le berbère a bénéficié de l'ouverture d'une licence d'enseignement à l'ENS d'Alger.

En matière de communication et des médias, la langue berbère bénéficie de deux institutions importantes à côté de la radio nationale (chaîne II) : ont été créées la télévision nationale en berbère (2009) et deux radios régionales kabyles: Radio Soummam à Bejaia (2000) et Radio Tizi-Ouzou (2011).

Par ailleurs, dans la perspective d'accompagner le berbère dans son nouveau statut, plusieurs chercheurs, universitaires et militants, se sont impliqués dans ce processus d'aménagement de la langue. En matière de lexique, de nombreuses initiatives individuelles sont à l'origine de la publication de plusieurs terminologies dans différents domaines : juridique (Adghirni et al. 1996), informatique (Saad-Bouzefrane 1996), religieux (Nait-Zerrad 1998), code de la route (Ali Khalfa 2005), littérature, géologie (Kamel 2006), grammaire et linguistique (Berkai 2009, Boumalek \& Nait-Zerrad 2009), littérature (Bouamara 2007, Salhi 2012), médias (Ameur et al. 2009) et électrotechnique (Mahrazi 2011).

Cependant, en l'absence d'une institution officielle d'aménagement linguistique, ces tentatives individuelles et institutionnelles ${ }^{2}$ (centres de recherches et universités) de propositions de terminologies ont engendré de nouvelles structures syntaxiques non attestées dans l'usage traditionnel et des choix différents en matière de création néologique. En effet, dans le souci de répondre, dans l'immédiat et dans la précipitation, aux besoins des nouveaux domaines de l'usage moderne, de nouvelles structures syntaxiques, lexicales, sémantiques sont apparues.

Le projet de notre travail est le résultat d'une expérience d'une dizaine d'années dans le cadre de l'enseignement de la matière d'expression écrite et orale aux étudiants de licence en langue et culture amazighes à l'Université Mouloud Mammeri de Tizi-Ouzou. Au cours de notre recherche nous voulons étudier le passage du kabyle en tant que langue réservée à l'usage oral à de nouveaux domaines d'usage : la communication, l'enseignement et les différents domaines modernes.

La présente contribution s'articulera autour d'une analyse lexico-sémantique et syntaxique de la terminologie du code de la route d'Ali Khalfa (2005) et des

2 Il s'agit des institutions qui n'ont pas de pouvoir juridique pour imposer des choix en matière d'aménagement. 
journaux d'information en berbère (kabyle) en mettant en exergue les procédés utilisés lors de la création des néologismes et les caractéristiques syntaxiques de la langue utilisée. Le choix de notre corpus est justifié par la place qu’occupent le code de la route et le journal d'information dans la communication quotidienne des différentes couches des locuteurs (lettrés et illettrés).

Afin de répondre progressivement à nos différents questionnements, nous avons procédé à la comparaison de la langue utilisée dans ces publications ${ }^{3}$ et celle de l'usage pour voir le degré d'attestation des règles en question dans le kabyle de l'usage moderne. Pour mener à bien notre travail, nous avons émis l'hypothèse que les textes de l'usage moderne du kabyle sont caractérisés par des structures calquées sur la langue française ${ }^{4}$, et sur le plan de la création néologique et au niveau syntaxique. En effet, certaines créations néologiques par calque génèrent de nouvelles structures non attestées et incompréhensibles dans l'usage quotidien.

Notre travail est une étude contrastive entre la langue française et le kabyle. Dans notre analyse nous nous sommes basés essentiellement sur les travaux de Lionel Galand (2010), de Chaker (1983) et de Kahlouche (1992). Pour atteindre notre objectif nous avons divisé notre recherche en deux points essentiels. Le premier concerne l'analyse des procédés de création néologique utilisés dans le travail du code de la route. Le second consiste à analyser les structures syntaxiques employées par les journalistes dans les textes d'information.

\section{La néologie dans le code de la route : création ou traduction?}

À l'ère des nouvelles technologies de l'information et de la communication, la néologie commence à occuper une place importante et donne lieu à une créativité illimitée dans les langues, et ce avec la création de nombreux néologismes dans les nouveaux domaines d'usage (enseignement, communication, etc.). Le domaine de la création de nouvelles unités lexicales est divisé en deux aspects essentiels : La néologie est réservée aux procédés de

3 Les structures de base de la langue de l'usage traditionnel avant l'intégration des nouvelles structures.

4 Le calque sur la langue française est justifié historiquement depuis la colonisation française en Algérie. 
formation et les néologismes pour les résultats de la création d'unités lexicales. En revanche, pour distinguer la langue de spécialité de la langue commune, on emploie néonymes pour les créations dans la première. Les néologues s'accordent à dire que, globalement, les procédés de formation lexicale se répartissent en deux groupes essentiels, qui sont la néologie de forme et la néologie du sens.

\subsection{La néologie formelle}

La néologie formelle est un procédé qui se base sur l'adjonction d'un morphème à un terme qui existe dans la langue. En effet, la création lexicale peut s'appuyer sur la dérivation et l'affixation et la composition.

\subsection{La néologie sémantique}

Contrairement au premier procédé, la néologie sémantique attribue un autre sens à une unité lexicale qui existe dans la langue. En effet, ledit procédé donne à l'unité lexicale une nouvelle acception dans la langue.

\subsection{Analyse du corpus}

Comme nous l'avons signalé ci-dessus, nous avons analysé les procédés de formation utilisés par Khalfa (2005) dans son travail consacré exclusivement au code de la route en kabyle Angal n webrid, publié par le Haut-Commissariat à l'Amazighité. L'ouvrage, composé de 121 pages, est divisé en trois parties : tisuknent taratakt « signalisation horizontale , tisuknent taglawit « signalisation verticale» et tisuknent tifawt «signalisation lumineuse », suivies d'un lexique utilisé, composé de 327 unités lexicales. À la fin du document, l'auteur signale ses propositions qui sont au nombre de 30 unités lexicales, ce qui représente 9,17\% de la totalité des unités lexicales. Cependant, les autres unités sont empruntées à Mammeri dans son dictionnaire Amawal n tmaziyt tatrart, «Dictionnaire du berbère moderne ». Le tableau ci-dessous intègre les différentes créations d'unités lexicales présentées par l'auteur, avec leurs équivalences en langue française. (Cf. Tableau 1). 
Tableau 1. Les créations lexicales de l'auteur

\begin{tabular}{|c|c|c|c|}
\hline Unité lexicale & Equiv. en français & Unité lexicale & Equiv. en français \\
\hline akeddar & trottoir & azeryisem & apéritif \\
\hline akriniten & tricolore & iłisemarsiw & digestif \\
\hline akudimiran & temporaire & illusan & cycliste \\
\hline amanzabrid & autoroute & imbeddi $n$ wurraf & arrêt de bus \\
\hline amecburrqay & réfléchissant & meglanamud & sens inverse \\
\hline amesniazdukklan & transport en commun & nnigusider & surbaisser \\
\hline amesddallus & motocycle & tacernint & pneu \\
\hline amyager & intersection & tagalist & panonceau \\
\hline anamud $n$ tuzzya & sens giratoire & tamesọarit & pédale \\
\hline anuzay & tracteur & tasundart & marchepied \\
\hline aqarran & radical & tazentilist & demi-barrière \\
\hline asankay & piston & tugzirt & îlot \\
\hline astenagayu & casque & tutțift & portique \\
\hline asuyim & poste & usliganamud & double sens \\
\hline awelluagejday & maître-cylindre & zertisuknent & pré-signalisation \\
\hline
\end{tabular}

L'analyse des procédés de création lexicale adoptés par l'auteur montre que 23 unités lexicales résultent de la formation morphologique. En effet, l'auteur utilise plusieurs procédés :

\subsubsection{Dérivation}

Lauteur a recouru au procédé de la dérivation par la formation des unités lexicales par l'ajout de plusieurs affixes :

Cycliste $:$ allus + an $=$ allusan (adjectif)

Piston : as $+\mathrm{nk}=$ asankay (nom d'instrument)

Apéritif : azer + ayisem $=$ azeryisem

Véhicule : $\mathrm{ams}+\mathrm{ddu}=$ amsedduy

Présignalisation : zer + tisuknent $=$ zertisuknent

\subsubsection{Composition}

Dans ce procédé de formation, l'auteur recourt à la fusion de plusieurs unités lexicales pour en créer de nouvelles : 
Double sens : uslig (adjectif) + anamud $($ nom $)=$ usliganamud

Cyclomoteur : allus (nom d'action verbale) + amsedday (nom) = allusmedday

Autoroute : amanziraz (nom) + abrid (nom) = amanzabrid

Marchepied : tasunt (nom) $+\operatorname{adar}($ nom $)=$ tasundart (nom d'instrument)

Tricolore $: \operatorname{krad}($ nom de nombre) + ini (nom) $=\operatorname{akrini}($ nom concret)

Demi-barrière : azgen (nom) + barrière (nom) $=$ tazentilist $($ nom concret)

\subsubsection{Néologie sémantique}

Concernant le procédé de la création sémantique, l'auteur a pris quelques unités lexicales qui ont déjà un sens dans la langue en leur conférant de nouveaux sens :

Allus : dérivé du verbe ales « répéter ", l'auteur l'utilise pour le sens de cycle.

Tuțtift : dérivé du verbe țtef « s'accrocher, prendre », l'auteur l'utilise dans le sens de portique.

Suite à cette analyse des propositions ci-dessus, l'auteur s'est basé principalement sur les procédés morphologiques, notamment la dérivation et la composition, plus que sur le procédé sémantique. En revanche, ce dernier est utilisé pour choisir l'unité lexicale à adopter pour le terme à créer avant de lui associer le procédé morphologique (la dérivation et la composition) dans la création de la nouvelle unité lexicale.

\section{Les constructions syntaxiques modernes dans les journaux radiodiffusés}

Après avoir traité les procédés de formation des néologismes dans le cas du code de la route en kabyle, nous tenterons maintenant de mettre en exergue quelques structures syntaxiques utilisées dans les textes journalistiques et qui ne sont pas attestées dans l'usage quotidien et traditionnel.

\subsection{Le syntagme prédicatif $d$ win « c'est celui »}

Il s'agit d'un syntagme prédicatif composé de l'auxiliaire de prédication $d$ "c'est » et du prédicat non verbal win « celui ». Dans l'usage traditionnel, l'emploi du syntagme en question est réservéà un emploi du choix et de singularité. Dans 
l'exemple du proverbe: Tamețtut d tin (féminin de win) iherrezen « La femme est celle qui préserve», l'emploi du syntagme prédicatif nous a permis de distinguer et de définir la femme en kabyle. Cette singularité est définie par l'emploi de la forme participiale du verbe qui suit le syntagme prédicatif. Cependant, nous avons relevé des emplois différents dans les textes journalistiques. Dans l'exemple: Argaz d win iruhen yer Tizi-Uzzu «L'homme c'est celui qui est parti à Tizi-Ouzou ». En réalité, l'auteur veut simplement dire que «l'homme est parti à Tizi-Ouzou» Argaz iruh yer Tizi-Uzzu..

\subsection{La forme passive}

Le berbère est caractérisé par un morphème du passif $(t w w, m, n)$ affixé au verbe. Son utilisation rend le verbe intransitif. Dans l'usage traditionnel le sujet devient patient. Yettwaker « il a été volé ». Dans cet énoncé nous nous ne pouvons pas savoir qui a fait l'action. Cependant, dans l'usage moderne, nous avons enregistré des structures phrastiques non attestées dans l'usage quotidien. Dans l'exemple yettwaker syur ameddakel-is « il a été volé par son ami », l'agent de l'action est explicité dans la phrase. Cette structure est en réalité calquée de la forme passive de la langue française, où le complément d'agent est néanmoins rare.

\subsection{L'usage abusif du nom d'action verbale à la place de la forme verbale}

Le morphème $M y$, préfixé au verbe, admet qu'un indice de personne pluriel dont l'expansion référentielle ${ }^{5}$ peut toujours être décomposée en deux ou plusieurs participants. Ces derniers effectuent et subissent simultanément le procès. (Chaker S. 1983 : 314). (12) msefhament «Ils se sont entendus» dans cette phrase, c'est le morphème $M y$ qui a assuré la réciprocité du procès des deux participants. Yella-d umsefham gar snat $n$ tmura, "Il y a eu un accord entre les deux pays». Dans cet énoncé, le journaliste voulait dire que les deux pays se sont entendus. Dans l'usage traditionnel du kabyle, le morphème de réciprocité permet à lui seul d'assurer simultanément le procès des deux participants. En revanche, dans ce cas, il s'agit d'un calque d'une construction à partir de la langue française, structure qui n'est pas attestée dans l'usage traditionnel du kabyle. En revanche, le journaliste peut recourir aux structures attestées dans l'usage en disant simplement : msefhament snat $n$ tmura « Les deux pays se sont entendus ».

5 Galand a adopté l'appellation de complément explicatif. 


\section{Conclusion}

Ce travail de défrichement peut être juste considéré comme une recherche préliminaire à un travail plus approfondi sur les procédés de formation des néologismes berbères dans les nouveaux domaines d'usage. Au terme de notre travail, nous avons constaté que le kabyle est très influencé par les structures de la langue française. Le locuteur ou le journaliste, dès qu'il se met dans une situation formelle pour traiter un domaine nouveau, fait recours directement aux structures de la langue française. De plus, les locuteurs kabylophones n'arrivent pas à transposer les structures traditionnelles de la langue dans les domaines nouveaux que le kabyle commence à intégrer. Cet état de fait pourrait créer deux situations linguistiques différentes, l'une pour l'usage courant, l'autre pour l'usage moderne. En somme, la situation pourrait être résumée ainsi :

- prépondérance de la création néologique par calque au détriment de la création sémantique ;

- privilège accordé à la création néologique au détriment de l'orientation des recherches vers les unités de la langue attestées dans l'usage traditionnel ;

- compréhension rendue difficile du fait des calques de structures syntaxiques du français.

En conclusion, nous pouvons dire que les changements qui caractérisent la structure syntaxique du kabyle ne sont pas dus à l'évolution progressive de la langue, mais, bien au contraire, il s'agit d'une déstructuration des anciennes règles morphosyntaxiques qui sont le résultat de l'influence des autres langues en présence, notamment la langue française.

«Les changements qui se manifestent au niveau syntaxique ne sont pas le résultat d'une évolution qui s'opère pour des raisons intrinsèques qui consisteraient en l'exploitation optimale des ressources qu'offre la langue mais ils sont en train de se concrétiser par imitation des langues dominantes comme conséquence d'une situation de concurrence linguistique qui est manifestement en défaveur du kabyle». (Achour, $2011: 632$ )

\section{Références bibliographiques}

Achour Ramdane, 2011, « Problèmes de construction syntaxique de la phrase en kabyle dans les usages moderne en situations formelles ", Parcours berbère, mélanges offerts Paulette-Galand Pernet et Lionel Galand pour leur 90ème anniversaire, BERBER Studies, Vol. 33, p. 621-632. 
Achab Ramdane, 2013, L’aménagement du lexique berbère de 1945 à nos jours, Tizi-Ouzou, Editions Achab.

Achab Ramdane et al., 1984, «Amawal n Tusnakt tafransist-tamaziyt (Lexique de Mathématique)», Tafsut, Tizi-Ouzou.

Adghirni Ahmed et al., 1996, Amawal azerfan. Lexique juridique français-amazighe, Rabat-Maroc, Tizrigin Imprial.

Ameur Meftaha et al., 2009, Vocabulaire des médias, Rabat-Maroc, IRCAM.

Berkai Abdelaziz, 2009, Lexique de la linguistique français-anglais-berbère. Précédé d'un essai de typologie des procédés néologiques, Tizi-Ouzou (Algérie), Éditions Achab.

Bouamara Kamel, 2007, Lexique de rhétorique (Amawal n tesnukyest), Alger, HCA.

Boudris Bélaid, 1993, Vocabulaire de l'éducation français-tamazight (tamawat n usegmi), Casablanca (Maroc).

Boumalek Abdellah \& Nait-Zerrad Kamal (coord.), 2009, Amawal $n$ tjerrumt (Vocabulaire grammatical), Rabat-Maroc, IRCAM.

Chaker Salem, 1983, Un parler berbère d'Algérie (Kabylie) : syntaxe, Aix-enProvence, Publications de l'Université de Provence.

Chaker Salem, 1991, Manuel de linguistique berbère I, Alger, Bouchène.

Chemime Mokrane, 1995, Allug n umzarur. Cahier de l'électricien, Tizi-Ouzou.

Galand Lionel, 2010, Regards sur le berbère, Milano, Centro Studi Camito-Semitici.

Kahlouche Rabah, 1992, Le berbère au contact de l'arabe et du français: étude sociolinguistique et linguistique, Thèse de doctorat d'État, Université d'Alger.

Kamel Said, 2006, Lexique amazighe de géologie, IRCAM, Rabat-Maroc.

Khelfa Ali, 2005, Angal n webrid, Alger, HCA.

Mahrazi Mohand, 2011, Lexique d'électrotechnique. Français-Tamazight, Alger, Éditions ENAG.

Mammeri Mouloud, 2008, Amawal $n$ tmaziyt tatrart (lexique de berbère moderne), CNRPAH, Alger.

Nait-Zerrad Kamal, 1998, Lexique religieux berbère et néologie : un essai de traduction partielle du Coran, Milano, Centro Studi Camito-Semitici.

Oussalem Mohand-Ouamar, 1999, "Quelques éléments sur les problèmes de l'expression en tamazight dans les usages modernes ». Timmuzgha $\mathrm{n}^{\circ} 02$, Alger Haut-Commissariat à l'A mazighité.

Saad-Bezfran Samya, 1996, Lexique français-anglais-berbère d'informatique, Paris, L'Harmattan. 
Salhi Mohand Akli, 2010, «Terminologie littéraire en amazighe », Asinag, $\mathrm{n}^{\circ}$ 4-5, IRCAM, Rabat, Maroc, p. 169-177.

Salhi Mohand Akli, 2012, Asegz awal amezzyan n tsekla (petit dictionnaire de littérature), Tizi-Ouzou (Algérie), Odyssée.

Touat Slimane, 1987, Lexique français- berbère de géographie, (manuscrit), Tizi-Ouzou. 